to orercome the obstruction, whioh was complete after the 14th, and she gradually eank and died on the 19th.

At the past mortem examination, the sigmoid flexure of the colon was found a solid mass of cancerous structure, the intestines above were distended with flatus and fluid froces, but presented no appearance of inflammation. The uterus and other organs were bealthy in structure, and the cancerous deposit appeared to be confined to the colon and the subcutaneous cellular tissue of the abdomen and chest.

CA8E II. Wm. Edwards, aged 58, admitted Feb. 23rd 1856, under Dr. Malden. He is a farm labourer, and has enjoyed good health, with the exception of occasional attacks of pain in the loins, which he looked upon as rheumatic, till two months ago, when he began to suffer from severe pains in the abdomen, especially in the lower part his stools were scanty and passed with difficulty, often mixed with blood: for this be went under the care of a surgeon, who told him he had inward piles, and gave him some medicine.

The abdomen is now enormously distended, very hard and tympanitic, painful, but not tender; the tympany is general, but if there is any difference, the left lateral region is less resonant than the others. He says that the bowels have not been moved since the 16th, and that on the 20th, 21st, and $22 \mathrm{nd}$, he vomited frequently. The urine is abundant, and the circulation is not affected. The compound extract of colocynth with senna was given in full and repeated doses with enemata, which were administered by a long tube; this passed frecly six or eight inches up the bowel, when it was stopped by some obstruction which did not yield to moderate pressure. The enemata were repeated several times to the extent of a pint or more, and some of them were very slightly coloured by fecal matter when returned.

Feb. 25th. As the purgative plan had produced no good effect, and the tongue had become dry, brown, and furred, with a rapid and feeble pulsc, great anxiety of countenance and loss of sleep, with romiting of the contents of the stomach, calomel gr. i, c. opii gr. ij, 4tis horis were substituted, and brandy with beef-tea administered every two hours, the enemata being continued.

Feb. 26th. Vomiting very trifling, as he can take but little food, and there is no nausea except from the presence of food in the stomach; the romited matters, far from being stercoraceous, have not even been tinged with bile. He feels easier, and the countenance expresses less distress, but there is no action of the bowels, and the relief is evidently due to the opium. Pulse remains feeble, but is less frequent than yesterday.

Feb. 27 th. The persevering use of enemata has hitherto been followed only by very slight staining of the fluid when returned, but this morning a small quantity of fæcal matter was observed. It was evident, however, that he was fast sinking in spite of the liberal use of brandy, beef-tea, etc., and he died the same day.

Post mortem examination, Feb.28th. Peritoneum healthy; stomach and intestines distended with flatus and fluid fæces, but healthy in structure as far as to the junction of the sigmoid flexure of the colon with the rectum. There was a firm mass of cancerous deposit in the coats of the bowel, which had encroached on the cavity of the intestine, encircling it for about two fingers breadth; the stricture allowed the points of the fingers introduced at opposite ends to meet, and would not alone have completely obstructed the passage of the contents of his bowel, but on further examination there was observed a remarkable hypertrophy of the bodies of the last lumbar and first sacral vertebræ, extending chiefly to the left side, so as almost to form an exostosis, which, corresponding with the seat of disease in the bowel, by its pressure had doubtless completely stopped the passage. Less developed hypertrophy of the bodies of the vertebræ was observed in the lower part of the dorsal region, and was the only further morbid appearance noticed in the body.

REMARKS. In both these cases the gradually increasing constipation and dificulty of passing stools pointed out the progressire derelopment of a mechanical obaticle. In the first, there could be little doubt as to its nature, from the development of cancer in the external parts; and in the second, the acute abdominal pain, and the hæmorrhage from the borel, would, in the absence of any hæmorrhoidal cause, lead to at least a strong suspicion of malignant growth. The absence of inflammatory complication was indicated, among other things, by the nature of the romiting, neither urgent nor stercoraceous; the non-inflamed intestine bore the presence of its contents without being stimulated to inordinate action; and in the second case, the complete absence of vomiting for some days after the canal of the bowel was obstructed, pointed out the lower bowel as the seat of disease; the free secretion of urine confirmed this, and the obstruction to the passage of the enema tube six or eight inches from the anus, completed the diagnosis.

With regard to treatment, the relief of the obstruction by the formation of an artificial anus was in the first case quite inadmissible; and in the second, considering the loathsome result of the opcration eren if successful, the rery probably malignant nature of the disease, and, above all, that the stoppage was not queite complete, it was thought better to rely upon the other remedies, than to have recourse to a dangerous operation, which could at best have only given temporary relief.

\section{ESSEX AND COLCHESTER HOSPITAL.}

\section{CLINICAI OBSERTATIONS ON A CASE OF PARTIAL} PARALYSIS, WHICH YIELDED RAPIDLY TO TREATMEXT.

By P. Martin Duncan, M.B.Lond., etc., Physician to the Hospital.

JANOARY 5th, 1856. G. Glozier, a short, wrinkled man, aged 61 years, was admitted under my care, complaining of inability to grasp with his right hand, to move his right arm, and to walk steadily. He suffered from a dull pain in his head, had slecpless nights, and had lost flesh.

IIistory. He had good health up to eighteen years ago, when he had syphilis very severely: he had sore throat, nodes on the shins, and a necrosis of part of the frontal bone. Two years since, he was treated for Potc's fracture by one of my colleagues. His recovery was complete, and, at the present date, no shortening is observable. Of late, starvation in a degree has weakened him: he has had plenty of bread, but meats and such like are strangers to him. About two months ago, he suffered from "pins and needles" in his right tingers; this symptom was speedily followed by a sensation of numbness in the fingers and arm, and loss of power soon supervened. He noticed, shortly afterwards, pain in the head; it commenced at night, prevented his sleeping, and affected his spirits. The right leg then began to lose power. He has taken little or no medicine, has had constipated bowels, but has not vomited. There is no history of rheumatic fever; he has no perspirations; and the man can remember no injury to his head or spine.

Present Condition. Is a short sturdy built man, has iron grey hair, no arcus senilis, but a very wrinkled face. He cannot see well with his right eye; but its pupil contracts equally with that of the left eye, and there is no cataract. There is no facial paralysis; his tongue is protruded properly, and with force; and his enunciation is distinct. There is a large scar orer the frontal sinus, but no pain is produced on pressure. He complains of constant pain, aggravated at night, in the left side of his head, in front of the parietal eminence; but pressure does not affect the intensity, or cause the pain. He has never had a fit of any kind, and there is no giddiness; but his memory is not so good as it was a few months since, and he says that he is always thinking about rery absurd and curious things. He 
aleeps beadly, hears well. and is very quiet and placid. The tongue is foul, and covered posteriorly with much light brown fur. His appetite is bad, and the bowels are constipated.

The right arm is thinner than the left (there is but little fat on his body); its muscles are less prominent, and it hangs loose, and is evidently in the way. The left hand grasps well, picks up small things, and is a healthy appendage to a healthy arm. The right hand is of the same temperature as the left, but no effort can close it with a very moderate grasp. He takes hold of one's hand with that odd eversion of the elbow which is so characteristic of a loss of power in the hand; and he cannot feel a pen or a knife, or direct his fingers to pick up small objects. There is incompetency in rotation in the forearm; it is extended with difficulty, and its skin is numb. The upper arm is affected in a less degree. There is no aneurism, no enlarged gland in the axilla, no periosteal disease, to press upon the brachial plexus; and he has not used a crutch.

When he is perfectly still, and half asleep, his right arm "jumps"; this subjective action has excited his wonder: and titillation over the back of the right hand and in the palm induces very active reflex contractions. There is no permanent contraction of the muscles of the affected arm; but they are irritable, and in anything but an anæmic condition.

The viscera appear to be healthy.

The right leg does not answer to his will as well as it used; but his unsteady gait is caused by his walking too much on his heels, and cver looking forwards in the course of his steps. He cannot walk comfortably with his head erect; he feels his way with his toes, and is evidently diffident of his powers of keeping up. There are no subjective morements in the right lower extremity, and there is no loss of sensibility. Ưrine as it has been for years, usually pale, and now and then staining the pot. Pulse 84

Diagnosis. From the age, the pain in the left side of the head, the loss of memory, and the sleeplessness, it appeared reasonable to infer a certain amount of perverted or defective nutrition in the superficies of the left lobe of the brain. The tingling, numbness, and subsequent loss of power in the right arm and hand-the side opposed to the seat of the cerebral pain-would seem to refer to a diseased condition of the corpus striatum of the left side. The amount of disease was supposed to be small, on account of the restriction of the paralysis to the nerves of the right upper extremity; and it appeared to me to be reasonable that acute softening of some of those fibres which connect the grey peripheral matter with the corpus striatum might have occurred. It was doubtful, however, whether acute softening of a portion of the corpus striatum, in combination with a disease of the superficies and of the meninges beneath the seat of the pain, and produced probably by the syphilitic taint, could account for the loss of sensation in the upper extremity, and the unsteady walking. The legs could be moved well enough when the patient was in his bed, but there was a certain difficulty in the association of the muscular movements to producc a good and firm walk. His peculiar gait struck me from the first as being cerebellar. The gradual numbness and loss of power to a certain degree only, and the reflex phenomena, referred the disease to the spinal cord; but the cerebral symptoms could not be over-estimated.

Although not disposed, from the conflicting symptoms, to localise the seat of the disease, I attributed it to the results of syphilis affecting the general nutrition of the brain; and I determined to try, after correcting the state of the bowels, the effect of long continued small doses of bichloride of mercury.

January 5th, 1856. Sumat pil. hydrarg. gr. V nocte maneque.

R Mag. carb. gr. $x$; mag. sulph. 3 ij; aq. menth. pip. そjij. Fiat haustus omni mane sumendus.

Jan. 6th. Well purged this morning. Pergat.

Jan. Fth. Bowels well open; tongue cleaner. Omit medicines.
Sumat pilnl, rhei comp. gr. $v$ omni nocte.

B Infus. quassix $¥ j$; hyd. bichlor. gr. 1-16. Fiat haustus ter die sumendus.

Jan. 10th. Much the same. Appetite better; pain in the head not so great, but his sleeplessness is great. Pergat. Jan. 12th. Still sleepless. Pergat.

Jan. 15th. Has improved, according to his own account, in the ability to grasp; but no very evident difference is obserrable. He says that he is always heavy for sleep, but that he cannot sleep from the "worry" of his thoughts.

Bx I'il. saponis comp. gr. v. Nocte sum. pro re natâ. Cont. mist. et pil. rhei.

Jan. 18th. Has slept well, having taken the opiate every night. Pergat.

Jan. 20th. There is a sensible amendment in his gait, and in the grasping power of his hand. His appetite he declares to be better than it has been for months, and he says that he no longer grows thin. Pergat.

Jan. 23rd. Improvement. The opium has lost its effect. To take one-third grain of morphia every night. Pergat.

IIe continued taking the bichloride until the 10th Feb.; on that day I could not detect any remains of his paralysis, and he could pick up his knife and fork, grasp firmly, and do the work in the ward. The subjective movements have left him, and his nights are better. His gums are slightly spongy, but there is no mercurial fotor.

Feb. 10th. He complains to-day of pain in his right sciatic notch. Omittatur hyd. bichloridum.

Bo Misturn camph. $\xi_{j}$; potassæ bicarbon. gr. $x$; vini se minum colchici mxv; morphiæ hydrochlor. gr. $\frac{1}{2}$. Fiat haustus omni nocte sumendus.

Feb. 26th. His rheumatic pains have left him; his appetite is cxcellent; and he has gained strength and weight.

B Potasse bicarb. gr. $x$; infusi quassix 3 . Fiat haustus ter die sumendus.

Feb. 28th. Quite well.

Fcb. 29th. Discharged cured.

REMARKS. The value of the gradual introduction of mercury, without its being allowed to pass on to salivation, was shown by the result of the case; and the relation between the two symptoms of pain and of paralysis was decided to be a close one by the excellent influence of morphia. The sensation returned with the ability to move; and the reflex actions ceased, as regards their intensity, as volition regained its sway. The return of appetite, of good spirits, and the loss of the general sense of misery, in this case, is an example of what has often been presented to $\mathrm{my}$ notice - of the tonic influence of mercury: tonic, of course, in a secondary sense; its primary action being expended upon a specific and most depressing poison.

\section{OHigimal Communiations.}

\section{NOTES ON THE PATHOLOGY AND TREATMENT OF ULCERS OF THE LEG.}

By Jous Kent Spender, Esq., Surgeon to the Eastern Dispensary, Bath.

Mr object in the following papers is to give a concise summary of the present views of the pathology and treatment of ulcers of the leg, and also to offer suggestions which may contribute to a better solution of the theory and practice of this disease.

Anybody who will take the trouble to attend the outpatient rooms of a hospital at once learns the extent to which ulcers of the leg prevail, and the obstinacy with which they resist treatment. Now these two facts comprehend the great points of $m y$ present inquiry: Why is the disease so frequent? and why is it with such difficulty amenable to the means adopted for its removal?

That the legs are more liable to ulcer than any other 